\title{
El techo de cristal en la industria hotelera de Tenerife (Islas Canarias): el acceso de las mujeres al liderazgo en el sector
}

\author{
Sara García Cuesta* \\ Universidad de La Laguna (España) \\ Francisca Galante Lorenzo** \\ Vicenta Poveda Verdejo**** \\ Investigadores independientes
}

\begin{abstract}
Resumen: Este artículo pretende acercarse a uno de los fenómenos de persistencia de la desigualdad de género en el trabajo como es el techo de cristal desde el ámbito concreto del sector hotelero de Tenerife. Se trata de uno de los resultados de un proyecto realizado desde la colaboración entre el Cabildo de Tenerife (FIFEDE) y la Federación de Hoteles de Tenerife (AShotel) en 2016-17. Se han identificado en este espacio económico de producción y empleo local los principales obstáculos con los que se encuentran las mujeres para acceder y permanecer en los puestos de media y máxima responsabilidad.
\end{abstract}

Palabras Clave: Turismo; Techo de cristal; Sector hotelero; Islas Canarias; Género; Igualdad laboral.

The glass roof in the hotel industry of Tenerife (Canary Islands): women's access to leadership in the sector

Abstract: This article aims at approaching the subject of the "glass ceiling", and the persistent phenomenon of gender inequality in the labour market, as shown through the analysis of the hotel sector in Tenerife. The research has been carried out in the context of a joint project with the Cabildo de Tenerife (FIFEDE) and the Hotel Federation of Tenerife (AShotel) in 2016-17. It has focused on detecting and identifying the main obstacles women face when trying to gain access to medium or high-ranking positions or managerial posts in this area of economic production, a niche for local employment.

Keywords: Tourism; Glass ceiling; Hotel sector; Canary Islands; Gender; Labour equality.

\section{Introducción}

Presentamos aquí algunos resultados relevantes del Estudio sobre el Techo de Cristal en la Industria Hotelera de Tenerife realizado de mayo de 2016 a mayo del 2017. La investigación fue financiada por La Fundación para la Formación, Empleo y Desarrollo Empresarial del Cabildo de Tenerife (FIFEDE) en colaboración con la Asociación Hotelera y Extrahotelera de Tenerife, La Palma, La Gomera y El Hierro (ASHOTEL). Se pretendió detectar y analizar rasgos y posibles factores de permanencia del conocido fenómeno del techo de cristal en el ámbito concreto de los espacios hoteleros de Tenerife. El techo de cristal, cuyos rasgos principales se presentarán en el epígrafe dedicado al marco teórico, básicamente pone de manifiesto la ausencia o muy reducida representación de las mujeres en los puestos de liderazgo en cualquier sector.

Los objetivos del proyecto localizaron tres interrogantes: ¿Cuáles son los factores que influyen en la persistencia del techo de cristal en el sector hotelero de Tenerife? ¿Qué dificultades específicas están

\footnotetext{
Profesora contratada doctor tipo 1 en el Dpto. de Sociología y Antropología de la Universidad de La Laguna, Espana; E-mail: sagarcia@ull.es

** Universidad de La Laguna, Investigadora independiente; E-mail: vicen_p@hotmail.com

*** Universidade de La Laguna, investigadora independente; E-mail: franigalante@gmail.com
} 
teniendo las mujeres para acceder, mantenerse y promocionar en el empleo en este sector? ¿Qué medidas se pueden tomar para reducir la brecha de género en los puestos directivos de los hoteles en Tenerife?

Estas finalidades orientaron una metodología multiestratégica que ha generado diversas categorías de datos y que será descrita en el epígrafe metodológico. La información obtenida ha permitido la aproximación al fenómeno laboral del techo de cristal en el sector hotelero de la isla, dibujado por una aproximación estadística, en primer lugar. A su vez, la información cualitativa obtenida a través de la realización de dos campos de entrevistas y el análisis del Convenio Colectivo, ha facilitado mostrar las dificultades específicas que las mujeres presentan en su progresión de carrera.

El resultado final del estudio incluyó una dimensión práctica, al generar recomendaciones orientadas a disminuir los obstáculos específicos que dificultan la presencia de mujeres en los puestos de dirección en los hoteles de Tenerife. Estas propuestas pueden ser consideradas en el diseño de un futuro modelo equitativo de gestión, que facilitaría las transformaciones organizacionales requeridas. Se mejorarían así las condiciones para la igualdad de oportunidades y el aprovechamiento de las capacidades y recursos humanos, disminuirían las barreras de género y mejoraría el impulso de las mujeres en los puestos más elevados del sector hotelero.

\section{Marco teórico}

La ausencia de paridad en los puestos de responsabilidad se relaciona con la existencia de barreras organizacionales en las carreras profesionales, además de otros obstáculos que, de manera específica, describen el contexto social y personal de estas mujeres. La expresión "Techo de Cristal" representa una brecha de género situada entre los puestos de liderazgo y el resto en la inmensa mayoría de las profesiones, organizaciones laborales y otros escenarios jerárquicos. La metáfora alude al material transparente, connotando que no son barreras formales y explícitas las que frenan la llegada de las mujeres al liderazgo. Tampoco leyes ni códigos civiles, sino factores diversos, de carácter más escurridizo, los que inciden en dificultar el desarrollo profesional de las mujeres en los diversos sectores de actividad (Burin, 2008; Wirth, 2002).

Además de la metáfora de techo de cristal, las ciencias sociales han acuñado en los últimos años otras expresiones para señalar las diferentes limitaciones con las que se encuentran las mujeres en el desarrollo de sus trayectorias profesionales. Por ejemplo, el "laberinto de cristal" representa las barreras a lo largo de la trayectoria profesional. Se localiza incluso una diferencia inicial en las trayectorias, identificada como "suelo pegajoso" (Eagly y Carli, 2007). Las carreras profesionales de las mujeres señalan más sobrecualificación y menores ingresos inicialmente, así como mayor permanencia sin promocionar, a pesar de contar con formación y experiencia laboral similares a las de los hombres y, en algunos nichos profesionales, más años de trayectoria (Smith, 2002; Mitra, 2003; García Cuesta et al, 2017). Una última representación gráfica, "el techo de cemento", incide en destacar los frenos autoimpuestos por las mujeres, implicados en el rechazo a la promoción a puestos directivos.

Mientras que el techo de cristal apela especialmente a las barreras externas del entorno social y organizativo, también denominadas objetivas, que se encuentran las mujeres en sus lugares de trabajo, el techo de cemento presenta una doble dimensión subjetiva en la que se implican la agencia y las autolimitaciones, bien estratégicas, bien asociadas a peores autoatribuciones: a) el rechazo de algunas mujeres a la promoción a puestos directivos más rígidos y exigentes, en previsión de las dificultades que puedan encontrar para conciliar su vida personal y laboral; y b) el prematuro abandono de las mujeres de los cargos directivos, asociada a la mayor presión que se ejerce sobre ellas (Chinchilla, Poelmans y León, 2005). Finalmente, se detecta la influencia de la construcción diferencial de expectativas y atribuciones que hombres y mujeres sienten sobre sus capacidades, aprendidas estas ya desde la infancia (Sáinz, Pálmen y García Cuesta, 2011).

Los aspectos brevemente señalados concluyen en un resultado final negativo para las mujeres, en términos de trayectorias de carrera y de acceso al liderazgo organizacional. La doble dimensión de la segregación vertical representada por los conceptos de techo de cristal y cemento permite identificar así barreras externas (en las organizaciones), internas (autoatribuciones) e interaccionales (asociadas a roles y responsabilidades diferenciales y a estrategias adaptativas) (Sáinz y Müller, 2018; Cambriles, et al., 2007; Ramos, et al, 2003). Una notable complejidad de factores influye a lo largo de la vida de las trabajadoras de manera exclusiva, incidiendo en sus oportunidades, decisiones laborales y en sus trayectorias profesionales. El goteo de efectivos acaba poniéndose de manifiesto en los puestos de liderazgo, profundamente ausentes de la participación de las mujeres. 
Figura 1. Identificación de factores que explican el Techo de Cristal

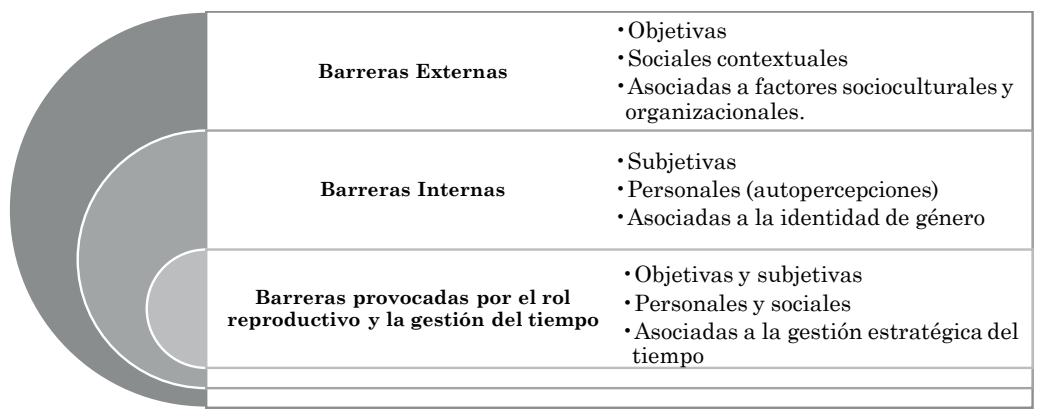

La muy limitada presencia de las mujeres en los puestos directivos ha sido puesta de manifiesto en numerosas investigaciones y es significativa en todas las estadísticas especializadas a nivel internacional (European Union, 2017; ILO, 2015). A pesar de ciertas mejoras experimentadas en los últimos años, asociadas al fuerte incremento de las cualificaciones educativas y profesionales de las mujeres en la OCDE, todavía hoy se mantiene la infrarrepresentación de las mujeres en los puestos de liderazgo económico y político.

En un marco del relevante impacto en el PIB y en el empleo, el sector turístico en Canarias es uno de los entornos laborales con mayor representación femenina. Un rasgo, por otra parte, extrapolable al sector turístico a nivel internacional. Pero este sector no es ajeno al techo de cristal ni a otros fenómenos de desigualdad de género en las organizaciones. Las investigaciones sobre turismo han incorporado muy tímidamente la perspectiva de género, de forma muy incipiente en sus análisis del sector laboral y en los relacionados con el turismo como fenómeno social, económico y de consumo (Segovia Pérez, 2013, p.4; Sigüenza, 2011, p.104; Fuller, 2015).

Estudios pioneros en perspectiva de género en el análisis del sector turístico como los referidos en el anterior párrafo apuntan un contexto de poca cualificación y desarrollo de capacidades competitivas en general, donde persiste una fuerte segregación laboral tanto horizontal (ocupacional) como vertical (a nivel jerárquico), especialmente en el subsector hostelero. Se detecta una clara segregación de la presencia masculina y femenina entre departamentos, la división sexual del trabajo en el reparto de las tareas y la presencia del techo de cristal: la menor presencia de las mujeres en los niveles de media y máxima responsabilidad (Segovia Pérez, 2013; Huete et al, 2016).

No obstante, los últimos años han mostrado un relevante empuje protagonizado por las mujeres en el sector del turismo, y en el subsector de la hostelería en particular, donde casi el $50 \%$ de los puestos de trabajo fueron ocupados por mujeres en el año 2015 en Canarias (ISTAC, 2015). La presencia proporcionada de mujeres en el sector no puede ser identificada con una situación de igualdad: una buena parte de su empleo en los hoteles se vincula a roles y estereotipos femeninos: limpieza, cocina, servicio de bares y restaurantes. A su vez, las mujeres ocupan los puestos más precarizados y los salarios más bajos en este ámbito (Richter, 1995). La industria hotelera ha sido señalada por su contribución a la reproducción de roles tradicionales asociados a las mujeres, así como de mantenerlas en puestos de menos cualificación y valoración (Sinclair, 1997; Hunter and Watson, 2006). Otros estudios han descrito el empleo en el sector como una pirámide con una base muy feminizada y con pocas mujeres en su cúspide. "El empleo en el sector del turismo ofrece a las mujeres oportunidades laborales, pero los puestos de trabajo que ocupan son mayoritariamente los peores en términos de remuneración y reconocimiento social" (Huete et al, 2016, p. 74).

Para propiciar un cambio de tendencia respecto a esta imagen del sector turístico como un ámbito en el que persisten las desigualdades laborales de género, iniciativas como la que ha dado lugar a esta investigación han querido contribuir a localizar los problemas específicos que dibujan el mapa de las barreras que deben atravesar las mujeres, a fin de facilitar estrategias tendentes a hacerlas desaparecer.

\section{Metodología del estudio}

Localizar los rasgos del techo de cristal en los hoteles de Tenerife implica aproximarse al estado de la cuestión en la distribución del empleo en el turismo a nivel local, así como recoger la experiencia de 
hombres y mujeres con trayectorias representativas en el sector. La metodología para ello se planteó como sigue: a) análisis de la base de establecimientos alojativos turísticos asociados a ASHOTEL $(n=192)$; b) la realización de una encuesta ad hoc a hoteles $(n=20)$; c) el análisis de las posibilidades formales vinculadas a la igualdad y conciliación que permite el Convenio Colectivo; d) la realización de cuatro entrevistas semi-estructuradas a agentes claves, representantes de organizaciones del sector (1 hombres, tres mujeres) y, e) finalmente, el análisis de 27 entrevistas en profundidad a 14 mujeres y 13 hombres con cargos de responsabilidad en los hoteles de Tenerife, a fin de aproximarse a los elementos diferenciales en sus trayectorias y a sus representaciones de los factores de impacto en las mismas.

Los hoteles que forman parte del análisis están asociados a Ashotel, que facilitó el listado de sus establecimientos alojativos turísticos, incluyendo las variables y datos diversos que permitieron realizar un análisis descriptivo de los establecimientos alojativos turísticos de la isla, atendiendo a la relación entre el sexo de las personas que los dirigen. Además de permitir un análisis estadístico que representa a 192 hoteles de Tenerife, estos datos apoyaron otras fases de la investigación, como la encuesta específica para conocer la segregación de género en las actividades, y la realización de los dos campos cualitativos de entrevistas a agentes claves y personas con cargos de responsabilidad en los hoteles.

El análisis de los datos secundarios y primarios generados concluyó con la elaboración del informe de resultados y recomendaciones que fue presentado en junio de este año ante el Cabildo de Tenerife y Ashotel en el que se pueden consultar resultados exhaustivos y las cinco fases del estudio que se llevaron a cabo ${ }^{1}$. Esta investigación aporta un elemento relativamente novedoso frente a otras precedentes (Segovia Pérez, 2013), al incluir las narraciones de los hombres sobre sus trayectorias y experiencias. Así pudimos identificar elementos y retos comunes y diferenciales en el acceso a liderazgo, clarificando el escenario de persistencia del techo de cristal en el caso de las mujeres.

La selección de la muestra de personas en puestos de responsabilidad implicó aquellos cargos cuyas tareas suponen la toma de decisiones y la coordinación de un equipo de trabajo. Se definieron finalmente los siguientes niveles de responsabilidad: a) nivel máximo de responsabilidad: direcciones; b) media alta: subdirecciones, asistentes de dirección, jefaturas de los departamentos y responsables de compras de los hoteles, c) media baja: segundas jefaturas de departamentos y responsables de reservas. Los factores de diferenciación de la muestra que han sido considerados para el análisis en las entrevistas han sido de tres tipos: a) de carácter demográfico: edad y sexo, b) de tipo laboral: nivel de responsabilidad desempeñado y departamento, y c) según la tipología del hotel: número de estrellas y si pertenencia a una cadena, grupo familiar o bien, hotel independiente ${ }^{2}$. Las dificultades para la localización de ciertos perfiles dan cuenta de una primera conclusión sobre el escenario de segregación horizontal (división sexual del trabajo) y vertical (jerárquica) que existe en los hoteles en Tenerife.

Los hallazgos plantean la posibilidad de un modelo de gestión en el sector que pueda tener en cuenta diferentes aspectos no considerados aún. Se trata de factores que están suponiendo una desventaja para la participación de las mujeres en las jefaturas: algunos de ellos muy explícitos, otros más sutiles, pero que condicionan indirectamente el acceso diferencial al liderazgo.

\section{Resultados}

En primer lugar, el estudio detecta la presencia de techo de cristal y de segmentación horizontal en los puestos de responsabilidad de la mayoría de las jefaturas que incluye la muestra de 192 hoteles proporcionada por Ashotel.

En casi todas las categorías no se alcanza la representación igualitaria entre ambos sexos (60\%-40\%), quedando las mujeres infrarrepresentadas en los puestos de media y máxima responsabilidad en los hoteles de Tenerife. Si bien encontramos un 35\% de presencia de directivas de hotel y departamento y un porcentaje algo mayor (37\%) en los puestos de segundo nivel (subdirecciones, segundas jefaturas). Las representaciones son optimistas, pues se acercan al 40\%, el primer paso para la situación de paridad.

No obstante, este acceso se caracteriza también por una segmentación horizontal por departamentos de mujeres y hombres en puestos de responsabilidad. En departamentos con más mujeres en plantilla, los liderazgos cuentan con presencia de mujeres y hombres (departamentos de limpieza y pisos). En departamentos con fuerza de trabajo mayoritariamente masculina el liderazgo es enteramente masculino (mantenimiento). No aparecen en la muestra jefas en cocinas, restaurantes y bar, pero se observa un $50 \%$ de jefaturas femeninas en Recepción y Relaciones Publicas.

El número de estrellas de los hoteles, así como su tamaño están relacionados también con la mayor o menor presencia de mujeres en el liderazgo. Los hoteles más grandes y con más estrellas cuentan con muchas menos mujeres en el liderazgo. Solo aparece un $13 \%$ de mujeres en puestos responsables 
en categorías cinco estrellas, mientras que en los hoteles de una a tres estrellas, la representación de las mujeres en cargos está dentro de los márgenes igualitarios.

Gráfico 1: Resumen del porcentaje de mujeres en liderazgo en los hoteles de Tenerife
según criterios de número de estrellas, tamaño de hotel, categorías de departamento

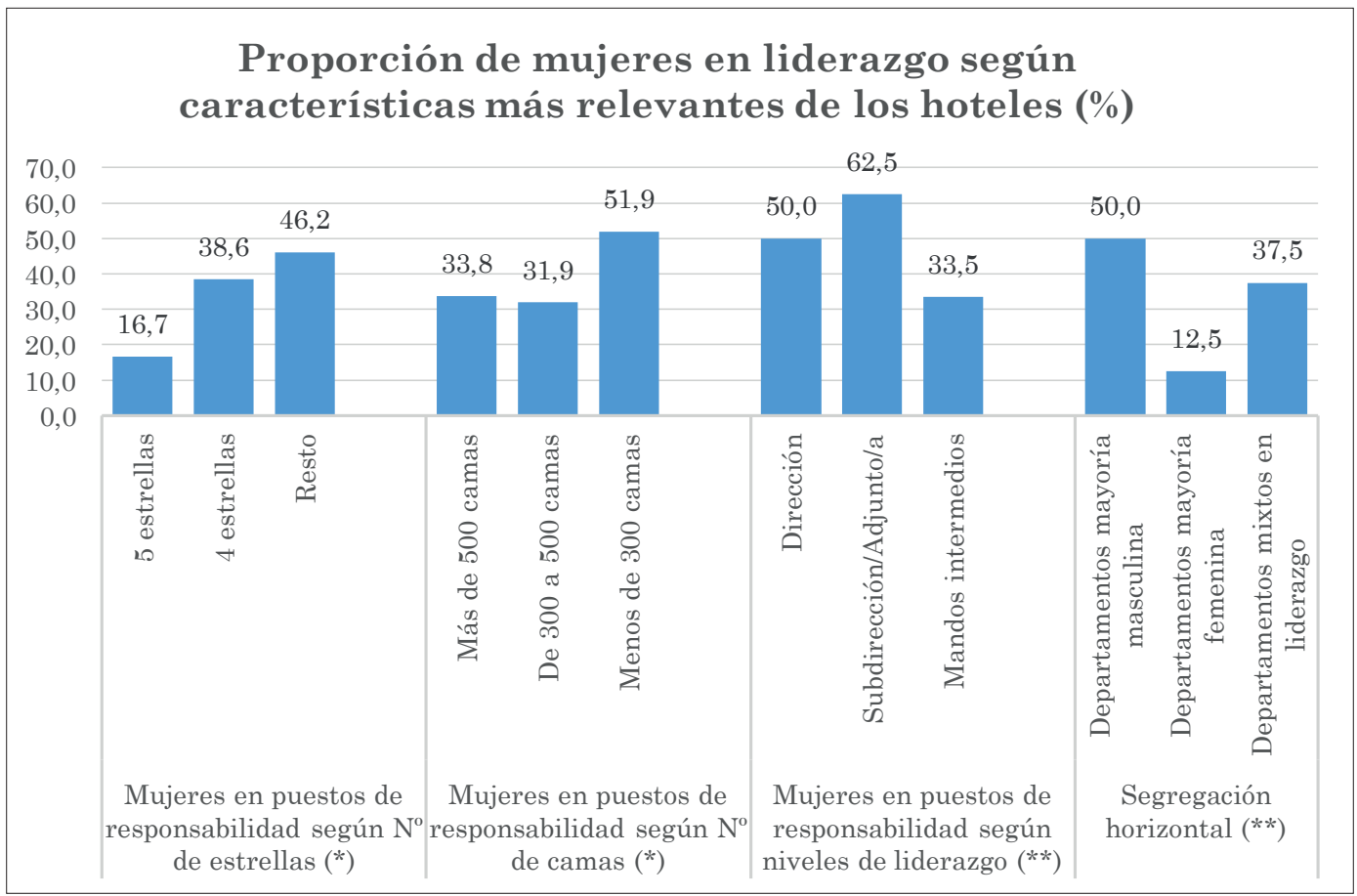

(*) Base de datos 192 hoteles ASHOTEL. Categoría directivas. (**) Encuesta a Hoteles (n=245 personas).

El impacto de la división sexual del trabajo aparece relacionado entonces con la distribución desigual del liderazgo femenino en el sector, poniendo de manifiesto las relaciones entre los aspectos de discriminación horizontal y vertical en el reparto del trabajo en los hoteles. El fuerte carácter generificado de la organización del trabajo entre hombres y mujeres en los hoteles escenifica además la distribución tradicional de roles domésticos, laborales y privados en las familias, puesto que los hoteles son espacios donde, al igual que en los hogares, es necesario realizar tareas asociadas a la reproducción (cocinar, limpiar, atender, cuidar, reparar, renovar) tanto como de producción (las tradicionalmente asociadas al empleo masculino).

Estas marcadas divisiones se reflejan también en las narraciones de hombres y mujeres entrevistadas, cuyos marcos de interpretación del sector tienen que ver con la asunción de este escenario. El techo de cristal, es decir, la infrarrepresentación de las mujeres en los puestos de liderazgo en los hoteles de Tenerife, se construye en trayectorias que reflejan circunstancias de promoción diferentes pero, sobre todo, desiguales entre mujeres y hombres. Las trayectorias de las mujeres son más heterogéneas (más fluctuaciones, cambios de trabajo, presencia de hitos y menor progresión lineal) y con mayor permanencia en los puestos intermedios (subdirecciones, adjuntas o asistentes de dirección) -lo que explica también su más alta representación en estos puestos intermedios-. Las mujeres presentan dilemas de conciliación y corresponsabilidad que los hombres no relatan y son conscientes de la persistencia de la división sexual del trabajo en los departamentos que muestran las cifras.

Una mención especial merece el fenómeno de las subdirectoras, situadas bajo el techo de cristal, cuyas experiencias y trayectorias contribuyen a explicar la infrarrepresentación femenina en las figuras de alto liderazgo en el sector hotelero de la isla: las mujeres permanecen más tiempo que los 
hombres en los puestos intermedios. En el sector hotelero de Tenerife esto se manifiesta en el puesto de subdirección, que ejerce de clásico tapón donde se acumulan más las mujeres que los hombres (62,5\% de subdirectoras). Como apunte meramente cualitativo, todas las entrevistadas que han pasado por este puesto perciben que su permanencia en el mismo ha sido mayor que la de sus compañeros. A su vez, las trayectorias de los entrevistados muestran menor tiempo en el puesto intermedio o incluso, un salto que prescinde del mismo.

“(...) Llevaba 6 años de subdirectora, y claro esperando el momento y sabes esto que intentas organizarte, todavía lo de ser mamá, yo quería pero todavía no porque claro primero tengo que lograr esa estabilidad que yo quería alcanzar, ese objetivo que me había puesto (...). Porque, si es verdad que los puestos de dirección siempre, bueno en las otras cadenas que he trabajado, siempre es asi, y sigue siendo, están un poco por hombría. A mí me ha costado mucho llegar ahí, aunque yo diga que bueno "tuve la suerte", me costó. Porque ahi a base de demostrar, demostrar, cuando eres mujer todavía lo tienes más complicado, lo siento, pero es así. (...) Te lo digo, casi que bueno, tenía que trabajar el doble. A mí se me llegó a dar el caso de tener un compañero que era subdirector también, con menos experiencia que yo y bueno, y había una promoción, ambos dispuestos isabes? (...) Y se lo ofrecieron a él incluso un comentario de, pero, era algo, era raro ino? (...). Después tuve la oportunidad de hablarlo con mi jefe (...) No supo darme argumentos, realmente no había, porque los resultados habían sacado tanto él como yo, entonces bueno, habrán dicho hombre, mujer, pues mira igual este que a lo mejor no nos va a dar problemas de que si se queda embarazado, porque es así. Supongo yo. Y de hecho esas conversaciones yo las he oído, qué lástima ino? He tenido compañeras en mi mismo puesto que han promocionado, sí, pero les ha costado pero... no te puedes imaginar. Y a base de estar ahí trabajando y vamos, al pie del cañón, como se dice, cada día, haciendo, no haciendo horas sino presentando todos aquellos informes... De alguna forma tienes que destacar żno? y sí he visto la diferencia ¿no? que a un hombre le cuesta mucho menos promocionar y lo he visto por eso lo digo así."

(Mujer, 30-45 años, Directora de hotel)

"O sea si estás en subdirección, te cambian a otro hotel como seguramente veréis en otras cadenas, te cambian constantemente del destino (...) te inventan puestos como subdirectora mega junior, subdirectora medio junior, simplemente junior, luego subdirectora mediana, luego subdirectora pues ya senior o empezando a ser senior y mientras tanto pues te sacan todo, toda la fuerza que tengas (...). Y ya dicen bueno esta ya es que lleva tantos años vale vamos a darle un hotelito este pequeñito que ya lo lleve. (...). muchas veces los chicos subdirectores que yo he conocido trabajan medio año y ya se van de director. Y en cambio las chicas pueden pegarse años y años y años y no significa que trabajen peor sino "zpor qué la vamos a cambiar si aquí está sacando el trabajo, está costando bastante barato por lo que hace y ni está exigiendo nada, diciendo ni pio?".

(Mujer, 30-35 años, Subdirectora de hotel)

"Tengo dos hermanas, de las cuáles yo soy el menor, (...) la mayor se dedica a lo que es la animación, el trato directo con los clientes y todo lo que son los jardines. (...) Mi hermana la del centro lleva conmigo el hotel (...). Entre los dos, sí, entre los dos. Cada uno sabe lo que está haciendo el otro, (...) Ella lleva lo que es todo el sector de alojamiento y pisos, vaya, y recepción. Yo por mi parte me dedico a lo que es, principalmente, a alimentos y bebidas y, cómo es, dirección técnica, cómo sería eso. (...) actualmente estoy como consejero delegado, sí, y luego mis dos hermanas como consejeras normales."

(Hombre, 40-50 años, Director de hotel)

"Primero firmé por 6 meses, luego por otros 6 y luego ya me hicieron el fijo, de la cadena. Incluso me cambiaron de categoría después de un año, en teoría tienes que esperar un poquito más todavía, creo que eran dos tres años o cuatro años tienes que estar en el puesto, algo así no sé muy bien como estaba especificado. Y me dieron la categoría superior."

(Hombre, 30-35 años, Subdirector)

Los rasgos de estos puestos de responsabilidad son la polivalencia, menores salarios frente a otros puestos directivos (incluidos dptos.), disponibilidad espacio-temporal, sobrecarga y problemas de conciliación. Se trata además de un perfil ajustado a la estereotipia de capacitaciones femeninas: capacidades multitarea, intermediación en conflictos, apoyo de plena disponibilidad a la dirección asumiendo de forma invisible tareas de la misma, importancia de la imagen femenina en segunda fila. Estos argumentos, cuando se ponen en valor desde el estereotipo, argumentan la permanencia de las mujeres en subdirecciones sin ascenso como figura de apoyo sobre-cualificada. 
"Pero es verdad que el salario de subdirección es muy bajo, (...) bastante más bajo que el salario de jefe departamental y el salario del director. ¿Entonces por qué las personas lo aceptan? Y muchas veces veréis que las subdirectoras la mayoría de las subdirectoras son chicas, por eso mismo, lo aceptan porque es un puesto, cuando te dan posibilidad de promocionar, de enseñarte, de trabajar, y currar hasta un puesto de directivo, (...) muchas veces los chicos subdirectores que yo he conocido trabajan medio año y ya se van de director. Y en cambio las chicas pueden pegarse años y años y años y no significa que trabajen peor sino: ipor qué la vamos a cambiar si aquí está sacando el trabajo, está costando bastante barato por lo que hace y ni está exigiendo nada, diciendo ni pio? (...) infravalorado, el trabajo de la subdirección y a nivel de salario. Porque si esta persona es la que saca todo el trabajo, reconócelo. Cómo va a mandar una subdirectora al jefe departamental que sabe que gana doble que ella, o triple."

(Mujer, 30-35 años, Subdirectora)

El análisis cualitativo revela también la existencia de laberinto de cristal, con dilemas específicos en las mujeres en puestos de dirección en los hoteles. Este fenómeno indica que el freno de las carreras femeninas no se produce al final de las trayectorias sino que estas van atravesando un recorrido que cuenta con obstáculos específicos ya desde los inicios. Las trayectorias masculinas relatan un recorrido más rápido, claro, con menos hitos de cambio, más homogéneo y libre de ciertos obstáculos que sí afectan a las mujeres. Corresponden con el modelo clásico de carrera, de trayectoria lineal, reconocimiento, progresivo prestigio, satisfacción con ingresos y recorrido, plena dedicación y sin interferencia de las responsabilidades de cuidados. Frente a estos recorridos propios de un modelo tradicional, las trayectorias femeninas relatan cambios más o menos bruscos en el recorrido, movilidad laboral no ascendente, más permanencia en la inestabilidad primero, luego en puestos intermedios, períodos de abandono o reducción del desarrollo de carrera que las sitúan en congruencia con los modelos periféricos de trayectoria detectados en otros estudios (Maranda y Comeau, 2005; García Cuesta, 2008).

Uno de los problemas que manifiestan específicamente las ejecutivas de estos hoteles es la dificultad de desarrollar la llamada doble presencia, que aparece aún asociada a sus relatos y asumida como responsabilidad propia de las mujeres -tengan empleo o no- en el marco de las estrategias familiares. Esto es, son ellas quienes manifiestan los conflictos cotidianos y de desarrollo de carrera que implica la doble jornada familiar de cuidados y laboral.

"Sí, porque yo, a ver yo no, es que tampoco tienes facilidades, no te es fácil. Primero, yo para buscar una guardería que cubriera los horarios que yo y mi pareja tenemos, me costó muchísimo (...) Tengo que pagar mucho para eso. Una guardería del estado no te cubre esos horarios por tanto tengo que pagar ese servicio aparte. (...) bueno que te voy a decir, es complicadísimo esto. (...) Bueno yo elegí eso, porque claro, también podía haber elegido pedir un permiso, una excedencia y quedarme, que tal. Oye que a mí me apetece mucho, que es mi hijo, pero es que tampoco me puedo permitir eso ¿sabes? Necesito trabajar y porque se trataba de adelantar un poquito para luego coger este puesto también."

(Mujer, 30-35 años, Directora)

“(...) cuando llegué sin ser madre, yo tenía un turno que era de 8 a 4, libraba sábado a domingo, y desde enero me están obligando a hacer guardias por las tardes, (...) no tengo guarderías para que me lo cuiden, es más complicado, (...) me dijeron que no. Que eso es lo que hay, que tengo que hacerlo, no le importa ni que yo lleve con este horario toda la vida, que yo organice mi vida y ser madre porque mi horario me lo permitía, mis guarderías, ¿̇sabes? (...) es que él también trabaja (...) ese es el problema, que nos ha trastornado la vida totalmente (...) emocionalmente me afecta bastante."

(Mujer, 40-50 años, Jefa de recepción)

La falta de medidas que posibiliten la conciliación entre la vida familiar y la laboral tiene efectos sobre las mujeres con cargos de responsabilidad en los hoteles. Ni las familias ni las organizaciones de trabajo han considerado estilos de gestión que no impliquen que sean las mujeres las que deban ocuparse casi en exclusiva de la doble o triple jornada. Así, son las mujeres con o sin hijos/as o personas a cargo quienes más señalan este problema como obstáculo y no sus compañeros varones.

“(...) Somos igual los hombres, somos iguales las mujeres, para mí, todos somos iguales. Pues si todos somos iguales, pues vamos a coger los mismos descansos, vamos a coger las mismas cosas y vamos a hacer lo mismo ¿vale? Y lo que yo trato es de buscar siempre que haya un sistema donde todos se toquen de la misma manera. Es decir, si me toca a mí un fin de semana descanso (...) porque en hostelería, no todos podemos descansar 
sábado y domingo. Por lo tanto hay que buscar una solución, pero la solución ¿cuál es?, es movemos todos, no hay descanso fijo, ni hay días fijos, ni hay puntos fijos, ni hay nada fijo. Todo se puede mover."

(Hombre, >50 años, Director de Departamento)

"P. ¿Cuando haces los turnos tienen en cuenta la situación familiar, si hay hijos pequeños o eso no lo tienen en cuenta?

R. No, eso no podemos tenerlo en cuenta. Aquí no, porque imagínate, no se le puede dar los fines de semana porque no tengan guardería a una y a las otras no. Porque todas trabajan igual. Entonces normalmente se hace un correlativo para que todas tengan sus días y ya ellas se organizan y también tengan con quien buscarlos, un día una urgencia que no puede venir se le tiene un poquito de cuidado, pero no puede haber preferencia."

(Mujer, 40-50 años, Subgobernanta)

Las mujeres en liderazgo que son madres describen el impacto en su gestión espacio-temporal cotidiana y en sus elecciones de carrera que les ha supuesto la maternidad. Una gran parte de ellas cuenta, no obstante, con el apoyo de otras mujeres (los conocidos fenómenos de abuelización, mercantilización o socialización de los cuidados a través de redes familiares o de amistad entre mujeres (Page, 1996). Los ingresos familiares les permiten la contratación de apoyo en el hogar y cuidados. Esto suaviza el conflicto en términos personales, laborales y familiares, pero desplaza el debate, en términos sociales, hacia otras mujeres trabajadoras.

"Entonces lo que me hizo dudar es eso, el miedo de decir, un niño tan pequeñito, cómo me voy a organizar, mis horarios, tengo horario partido, tengo que estar hasta tarde en el hotel. Pero bueno, ya eso ha pasado, cuento con la ayuda de mi madre, porque si no tampoco creo que hubiera sido capaz, no lo sé. Y luego está la guardería también, que no lo he tenido que llevar todavía, ir a la guardería, pero bueno que está ahí ¿sabes? (...) Lo que ellos no entienden que yo renuncie a esto para encargar... entonces tengo el apoyo ese, yo será que tengo una familia muy unida y por ahí, lo tengo fácil, pero entiendo que sin ellos no sé qué hubiera hecho."

(Mujer, 30-40 años, Directora)

Frente a estas mujeres, las directivas sin hijos/as localizan la maternidad como un freno y optan por retrasarla o renunciar a ella. Se trata de mujeres con clara orientación a la carrera, que entienden que el desarrollo personal no tiene por qué implicar la maternidad/paternidad, o, al menos, no circunscrita a una etapa específica. Si bien el planteamiento de esta decisión como renuncia o retraso indica cierta frustración de metas vitales, que seguramente se plantearían de otras forma si existirán medidas de conciliación eficaces en las empresas y corresponsabilidad en las familias. Frente a las directivas (con o sin hijos), los hombres no refieren esta disyuntiva como problema cotidiano o a largo plazo. Tampoco señalan la incompatibilidad de su paternidad con el desarrollo de su carrera profesional, e incluso pueden identificarla como un elemento de motivación para el asentamiento laboral y refuerzo de la meta profesional.

No pocas de las personas entrevistadas han contado con apoyos a la conciliación basados en la delegación de parte de los cuidados en terceras mujeres que resultan muy relevantes para facilitar las trayectorias profesionales. Pero sobre estas cuestiones hablan particularmente las mujeres (localizando un conflicto personalizado en ellas), mientras que sus parejas no aparecen en sus discursos como corresponsables, sino como apoyos con un rol secundario en los cuidados.

"Yo pienso que es un problema cultural debido a que la mujer siempre se ha dedicado más a las criaturas, si tienes un matrimonio pues, siempre es la visión de que es la mujer la que se va a encargar del hijo, (...). Entonces es más difícil poder acceder a un puesto de responsabilidad, si ya la misma mujer pues dice si a mi me toca estas funciones para qué voy a estar correcta, (...) entonces (...) que se reparta los roles en casa, yo pensaría que debe de ir más bien por ahí. (...) tu va a mi casa y te das cuenta, mi mujer (...) no tenía estudios universitarios y yo he llegado a donde llegado, claro, también cuando yo la conocí no tenía estudios universitarios."

(Hombre, >50 años, Director de departamento)

"P. (...) concilias, por lo que veo, bien tu vida laboral con tu vida familiar...

R. Mi mujer dice que no, pero yo le digo que sí.” 
"Entonces el hombre es que ni le pasa por la cabeza que tiene que atender a sus hijos a partir de, no que atender si no qué hacer con los niños a partir de las 5 de la tarde. O sea el puzle ese de tengo que preparar la comida para mañana, tengo que llevarlo al cole, tengo que llevarlo al futbol, o sea eso, o sea vamos, en la mente de los hombres no cuadra, o sea. Pues los niños se van solos, yo que sé, alguien los llevará."

(Mujer, 30-40 años, Directora)

\begin{abstract}
"Si yo salgo de mi casa a las nueve de la mañana y vuelvo a las nueve de la noche, que voy a tener a una señora pagándola para criar a mi hijo, o sea, al final te planteas dos opciones: una es, o vuelvo a un puesto de menos responsabilidad, de recepción con un horario fijo de mañana de ocho a tres de jornada reducida; o la otra opción, que te planteas muchas veces, (...) me lo planteo ahora mismo, es que hasta que mi hijo tenga cinco años, claro, o dejas de trabajar y pides una excedencia, pero no creo que sea eso. Sabes, es como, a ver, al final, siempre han sido las mujeres las que se sacrifican por los hombres que van a los puestos de responsabilidad y demás, ¿no?"
\end{abstract}

(Mujer, 30-40 años, GEX Experience Manager)

"P ¿De quienes te llegan más solicitudes de permiso?

$R$-Ya sabéis la respuesta ¿no? Las mujeres, por supuesto.

$P$-Y los hombres ise nota la diferencia?

$R$-Se nota, se nota. De los hombres más bien lo que yo he notado es falta al trabajo por algo, por fiesta, bueno hombres, chicos, quiero decir. O bueno a lo mejor la enfermedad o bajas por algún, bueno. Permisos, como permisos de cuidar, las mujeres."

(Mujer, 30-35 años, Subdirectora de hotel)

"P-¿Quién reduce jornada laboral, por ejemplo?

$R$-Pues todas las reducciones que tenemos de jornada igual un noventa y tantos por no decirte un 100\% son las mujeres."

(Hombre, 40-50 años, Director de Departamento)

El sector hotelero no presenta medidas de conciliación que pongan de manifiesto un compromiso visible con la garantía de la igualdad de oportunidades en el trabajo entre hombres y mujeres. No se localizan medidas formales que favorezcan que tanto mujeres como hombres puedan atender la doble responsabilidad cotidiana entre la familia y el empleo. Tampoco hay incentivos específicos para que los hombres se sientan más inclinados a participar en los cuidados en sus familias, en modelos de corresponsabilidad. Los hombres y mujeres de la muestra asumen estos conflictos como una cuestión de redes domésticas a resolver en un contexto familiar, cuando no un problema exclusivo de las madres. Solo una participante de la muestra plantea la responsabilidad de la empresa en contribuir a la conciliación puesto que la falta de resolución de los problemas del reparto del trabajo de los cuidados genera desigualdades en los recorridos profesionales.

“(...) si quizás si la mujer tuviera un poquito más de apoyo por parte de las empresas en general, pues habría menos complicaciones o sea para compaginar la vida personal con la laboral, todo eso ayudaría teniendo además en cuenta que el peso familiar todavía lo lleva la mujer encima."

(Mujer, 30-40 años, Directora de hotel)

En el marco de los modelos tradicionales de carrera profesional y organización empresarial, se mantiene una cultura de la presencialidad que perjudica el ascenso de las mujeres y supone pérdida de recursos humanos valiosos, al no utilizar las nuevas cualificaciones y recursos profesionales TICs para optimizar eficientemente el trabajo. La plena disponibilidad sin horarios en la que se basa el modelo tradicional perjudica a las mujeres con altas cualificaciones que asumen doble presencia o renuncian a tener familia. Especialmente, porque tanto familias como organizaciones asumen los cuidados como responsabilidad personal de ellas.

El análisis subraya cómo las organizaciones asumen la asignación de los cuidados a las mujeres, en dos sentidos: a) entienden que son ellas las que desempeñan la doble presencia, asumiendo el rol de cuidadoras de hijos/as, personas mayores o con enfermedades y b) entienden que el trabajo de los 
cuidados es un problema personal que debe resolverse en escenarios doméstico- familiares. Como consecuencia de estos dos presupuestos, los cuidados se asocian simbólicamente a las trabajadoras y profesionales -que finalmente los desempeñan- y se entienden como problema personal de las mujeres.

Es importante destacar que el conflicto de la doble presencia no es exclusivo del sector hotelero de Tenerife. Se repite en todos los ámbitos profesionales. Solo algunas profesiones están modificando sus pautas hacia la corresponsabilidad y conciliación. Este camino invita a la innovación beneficiosa no solamente para avanzar en igualdad de oportunidades y hacia la equidad en los sectores laborales. También para comprometer a los hombres en los cuidados, y facilitar modelos de una paternidad más consciente, comprometida y cotidiana frente al modelo de padre ausente asociado al desarrollo de sus carreras profesionales.

El análisis del Convenio permitió comprobar de facto un marco formal de interpretación de los relatos que revela que el sector hotelero de Tenerife no cuenta con políticas activas de igualdad de oportunidades. Tanto la reglamentación como los discursos de representantes no incorporan planteamientos actualizados en lo referente a las políticas de conciliación. En este sentido, el análisis pone de manifiesto la falta de información sobre las discriminaciones que están teniendo lugar en el sector; así como el desconocimiento del papel activo y facilitador de las empresas respecto a la igualdad de oportunidades y la equidad. Un indicador relevante es que, al entenderse la conciliación como un problema personal de las mujeres, se identifica solo con ajustes horarios más o menos espontáneos que se les otorgan a ellas, entendiéndolos, además, como favores.

En los relatos generados por las entrevistas en los dos campos aparecen estereotipos de género que pueden estar dificultando el ascenso de las mujeres, su presencia y reconocimiento en áreas masculinas. No solamente prejuicios que asocian a las mujeres determinadas tareas y empleos. También aquellos otros que califican modos de carácter y capacidades diferentes y en escala de valor, entre hombres y mujeres, bien debidas a su supuesta naturaleza o a su aprendizaje cultural.

Los estereotipos se mantienen sin una adecuada política de Recursos Humanos para la selección y promoción. En este sentido, hay algunos avances de carácter limitado como los muy bien valorados programas de promoción interna y formación directiva en los hoteles de cadenas, un incentivo que anima a las mujeres al liderazgo. Además resulta positiva en términos de innovación e igualdad la mera existencia del Protocolo de prevención y atención del acoso sexual y por razón de sexo de Ashotel y otras diversas acciones de sensibilización desde el ámbito sindical. Sin embargo, si comparamos estas incursiones con el discurso mayoritariamente recogido en este estudio y con el escenario formal de avance e implementación de medidas de igualdad desarrolladas en otros sectores que movilizan grandes volúmenes de trabajo en la isla, el sector hotelero de Tenerife tiene un gran reto que plantearse en estos términos.

En este escenario de retos, el más relevante apoyo para el avance es, precisamente, el incremento reciente de las mujeres en puestos de responsabilidad. De hecho, otro de los resultados relevantes del estudio se ha apoyado en relacionar las posibilidades de los nuevos estilos de liderazgo en el sector hotelero con el impulso de las mujeres y también con la mejora de sus posibilidades de acceso al liderazgo en el sector (Ashotel, 2017). En este sentido, es necesario resaltar las aportaciones que las mujeres pueden hacer en las organizaciones. Estas se asocian más a los nuevos y deseables estilos de liderazgo, lo que no quiere decir que sean exclusivos de ellas ni que no haya mujeres que respondan a modelos más tradicionales. Si bien, sus discursos sobre el liderazgo se asemejan más a estos nuevos estilos, muy probablemente debido a que las trayectorias profesionales de las mujeres también han sido diferentes al modelo tradicional.

Los viejos estilos de liderazgo se caracterizan por estar burocratizados, basados en la rigidez de organización, con distancias jerárquicas y actitudes autoritarias, un modelo presentista y reproductor de la división sexual del trabajo. Estos estilos no responden a los avances sociales y ni a los nuevos perfiles y exigencias de una clientela que sí está actualizada. Frente a ellos, los nuevos estilos de liderazgo usan las TICs, se basan en la eficiencia (frente a la presencia) y la delegación (frente al control vertical). Las cualificaciones implícitas se relacionan más con la negociación que con la autoridad explícita, la adaptación de soluciones a los problemas, la conciencia sobre el peso de estereotipos, y el reconocimiento profesional a quienes resulten más adecuados a los puestos en condiciones y con garantías de igualdad de oportunidades. Los nuevos estilos de dirección evitan las discriminaciones en el entorno laboral y del servicio al cliente, favorecen el buen clima laboral y buscan soluciones de equipo para las cuestiones de conciliación, colectivizando las responsabilidades (Cuadrado, 2014).

Las organizaciones contribuyen al progreso social cuando incorporan diversidad de género en sus estilos, a través de criterios de equidad. Y se ven beneficiadas por esta diversidad, puesto que las mujeres 
presentan también excelentes cualificaciones profesionales y formativas que son sistemáticamente más valoradas en los procesos de selección curricular anonimizados, evidenciando el peso de los estereotipos en la valoración tradicional de los méritos. En ese sentido, visibilizar a mujeres líderes genera un efecto social positivo para el sector turístico de Tenerife: mejoraría la imagen de cada hotel en términos de innovación, igualdad, progreso, justicia y profesionalidad. El nuevo turismo exige además, diversidad de género y atención de calidad que evite reproducir los estereotipos sexistas y de todo tipo.

\section{Conclusiones y aportaciones}

Este artículo ha mostrado de forma sucinta algunas de las aportaciones más relevantes del citado estudio sobre el techo de cristal en el sector hotelero de Tenerife. El estudio ha permitido un análisis comparativo entre las trayectorias de hombres y mujeres en posiciones de liderazgo en un sector relevante de la economía, de impacto muy significativo en este territorio. Así mismo, el estudio aporta una aplicación diagnóstica local para una futura propuesta de mejora del sector, a través del desarrollo de un modelo piloto equitativo de gestión. Sus conclusiones operativas permiten dimensionar el fenómeno del techo de cristal en el sector y apuntar factores implicados en su persistencia. Se trata, finalmente, de una contribución al desarrollo de los nuevos estudios vinculados a las políticas de igualdad en el entorno canario.

Los datos de la muestra de hoteles de Ashotel en Tenerife subrayan inicialmente las mejoras en la representación de las mujeres en los puestos de liderazgo en el sector. Si bien, dan cuenta a su vez de distribuciones muy marcadas por la división sexual del trabajo entre los diferentes departamentos y entre los puestos de primer y segundo orden en la jerarquía. Se observa además como los hoteles más grandes y con más estrellas -generalmente de cadenas hoteleras- son los que presentan un techo de cristal más marcado, es decir, donde menos directivas encontramos.

$\mathrm{El}$ análisis de las trayectorias profesionales ha localizado resistencias a la dirección femenina en empresas y organizaciones relacionadas con las formas de trabajo, el modelo de carrera y el peso de los estereotipos de género. Estas resistencias, asociadas a estilos tradicionales de organización en el sector, constituyen barreras específicas para ellas. Además de las barreras externas, vinculadas a los rasgos del sector, también se han evidenciado la tendencia de autolimitación de las mujeres en el desarrollo de sus carreras. Se trata en este caso de barreras relacionadas con la percepción sobre sus posibilidades y capacidades en los entornos laborales y su menor orientación estratégica hacia el éxito laboral (definido este bajo parámetros masculinos).

Una tercera desventaja se une a los rasgos limitantes del sector hotelero y de las autopercepciones en desventaja de las mujeres de cara al liderazgo. Esta última tiene consideraciones políticas, pues está definida como barrera relacional, vinculada a la asignación a las mujeres de los roles del trabajo reproductivo tanto en las organizaciones laborales como en la doméstica. En el caso del sector hotelero de Tenerife, no hay medidas efectivas para que las empresas puedan contribuir a la resolución de los conflictos de la conciliación y la corresponsabilidad que dificultan las trayectorias femeninas hacia el liderazgo.

El conjunto de circunstancias genera un retardo importante en la esperada mejora sustancial en el porcentaje de mujeres en estos puestos, consecuencia del empuje formativo y profesional de las nuevas generaciones de féminas. Las entrevistas ponen de manifiesto como estas mujeres perciben menos oportunidades de promoción, están más en puestos peor remunerados y reconocidos, tienen funciones y responsabilidades por encima de las contratadas (sobre-cualificadas, subocupadas), pasan más tiempo en puestos intermedios, perciben que tienen que demostrar más y que se les reconoce menos. Sus trayectorias hasta el liderazgo se presentan, efectivamente, más largas y heterogéneas.

Las consecuencias de los tres tipos de barreras localizadas contribuyen en su conjunto a explicar las más complejas y lentas trayectorias femeninas, con más hitos de cambio y definidas por las mujeres (y no por los hombres) en términos de ciertas renuncias que solo ellas experimentan: bien la renuncia a la promoción -para cuidar a los hijos/as; bien la renuncia o retraso de la maternidad, por percibirla incompatible con el desarrollo profesional. Las directivas no asocian los problemas en sus recorridos con la desigualdad de oportunidades, cuya influencia no localizan abiertamente. Sin embargo, señalan la falta de apoyos de conciliación en su trabajo y el limitado alcance de la corresponsabilidad en sus hogares. En la descripción de la gestión cotidiana y las trayectorias de carrera, sus relatos remiten de facto a los citados dilemas -solo en el caso de ellas-. 
Las dimensiones de influencia de un entorno social que no permanece ajeno tampoco a la presencia de este tipo de barreras no se escapan a las consideraciones de esta reflexión. Una parte de las desventajas que tienen las mujeres en su acceso al liderazgo en general tiene que ver con modelos relacionales, estereotipos y circunstancias discriminatorias sobre las que es necesario actuar desde instancias públicas y privadas, desarrollando las posibilidades que plantean las normativas de igualdad.

En ese sentido, el sector hotelero de Tenerife puede contribuir enormemente al socavamiento de algunas de estas barreras. Especialmente aquellas denominadas externas, que corresponden a los modelos de organización basados en la división sexual del trabajo, al estilo de liderazgo rígido, al modelo tradicional de carrera presentista y sin ausencia de medidas de conciliación. La carencia general de información e innovación en igualdad hace recomendar la necesidad de diseñar políticas de organización del trabajo y de la promoción que faciliten la conciliación a hombres y mujeres y que comprometan a las empresas en esta corresponsabilidad. Se trataría de promocionar un modelo de carrera más actual, que permita adaptarse a las necesidades cambiantes de conciliación de las y los trabajadores. Estos nuevos modelos de trabajo se reflejarían también en novedosos estilos de atención y un cambio de imagen necesario para responder a las necesidades reales de la clientela y el servicio. Puesto que la clientela espera y valorará una atención acorde con el trato igualitario a hombres y a mujeres. Por ello, se subraya la importancia de incorporar la perspectiva de género en las organizaciones del sector, revisando los procesos de organización del personal, selección, promoción, formación y atención al cliente.

Por último, dado que se ha localizado un impulso en la emergencia de las mujeres líderes en el sector hotelero, sería muy positivo visibilizar a estas mujeres. Su imagen sin duda animaría a otras a reconocerse en estos referentes de éxito. La existencia de modelos de referencia y del mentorazgo femenino, contribuiría a romper las autoatribuciones negativas de las mujeres con respecto a sus posibilidades de éxito profesional. También localizaría este escenario hotelero como una interesante opción de empleo para las jóvenes cualificadas que aspiren a alcanzar el éxito profesional sin las barreras de sus antecesoras. Reducir las barreras localizadas hasta conseguir finalmente eliminarlas es posible si las empresas se animan a iniciar procesos innovadores que permitan reducir estar desventajas para las nuevas generaciones de profesionales del sector hotelero de Tenerife.

\section{Bibliografía}

Balbo Laura

1994. "La doble presencia”. En Borderías, Cristina, Carrasco Cristina y Alemany, Carmen (Eds.), Las mujeres y el trabajo. Rupturas conceptuales (pp.503-510). Barcelona y Madrid: Icaria-Fuhem.

Burin, Mabel

2008. "Las 'fronteras de cristal'. En la carrera laboral de las mujeres. Género, subjetividad y globalización”. Anuario de Psicología, 39 (1): 75-86.

Cambriles, Maricruz, et al.

2007. Diagnóstico y autodiagnóstico de barreras de género. Posicionamiento de la mujer frente al empleo.

Colegio de Psicología de Andalucía Occidental: Proyecto EQUAL "e-Andaluzas en la Sociedad red".

Iniciativa Comunitaria 2004-2007.

Chinchilla N, Poelmans, S. y León, Consuelo

2005. Mujeres directivas bajo el techo de cristal. International Center of Work and Family.

Cuadrado, Isabel

2003. “Emplean hombres y mujeres deferentes estilos de liderazgo? Análisis de la influencia de los estilos de liderazgo en el acceso a los puestos de dirección”. International Journal of Social Psychology 18 (3): 283-307.

European Union

2017. Report on equality between women and men in the EU. Brussels: European Commission

García Cuesta, Sara; Poveda Verdejo, Vicenta; Galante Lorenzo, Francisca

2017. Estudio sobre el techo de cristal en la industria hotelera de Tenerife. Tenerife: Ashotel-Fifede.

García Cuesta, Sara

2008."Mujeres en trayectorias periféricas de carrera: las abogadas en doble presencia". Revista Clepsydra

(7): 43-70.

Huete, Raquel; Brotons, Matilde y Sigüenza, María del Carmen

2016. "La desigualdad entre mujeres y hombres en el sector hostelero español". Revista Estudios y perspectivas en Turismo 25: 73-87. 
International Labour Organization (ILO)

2015. Women in Business and Management Gaining Momentum Global Report. Geneve: ACT/EMP Bureau for Employers' Activities.

Lupano Perugini, María Laura

2009. Nuevas metáforas acerca de las mujeres líderes. Psicodebate 9. Psicología, cultura y sociedad.

Norma Fuller

2010. "Lunahuaná, un destino turístico. Transformaciones en la composición social, economía familiar y relaciones de género". Pasos, Revista de Turismo y Patrimonio Cultural 8 (2): 293-304.

Maranda, Marie-France and Comeu, Yvan

2000. "Some contributions of sociology to the understanding of career (pp. 37-53)". En Collins, A. y Young R.A., The Future of Career. Cambridge: Cambridge University Press.

Page, Alvaro

1996. Los usos del tiempo como indicadores de las discriminaciones entre los géneros. Madrid: Instituto de la Mujer, Colección Estudios 46.

Ramos, Amparo, Barberá, Esther y Sarrió, Maite

2003. "Mujeres directivas, espacio de poder y relaciones de género". Anuario de Psicología 34 (2): 267-278.

Sáinz, Milagros y Müller, Jörg

2018. "Gender and family influences on Spanish students' aspirations and values in stem fields". International Journal of Science Education 40(2): 188-203.

Sáinz, Milagros; Pálmen, Rachel y García Cuesta, Sara

2011. "Parental and secondary teachers' perceptions of ICT professionals, gender differences and their own role in the choice of studies". Springer: Sex Roles 1 (59): 150 - 180.

Segovia Pérez, Mónica (dir.)

2013. Mujer y techo de cristal en el sector turístico. Madrid: Estudios e Investigaciones del Instituto de la Mujer y para la Igualdad de oportunidades.

Sigüenza, María Carmen

2011. "La mujer en el subsector del alojamiento. El caso de la Costa Blanca (Alicante, España)". Investigaciones Turísticas, 2: 102-119.

Sommers, Carol

2017. From the Glass Slipper to the Glass Ceiling: A Study of Career Progression of Working Mothers in Middle to Top Management. Prescott Valley, Arizona: ProQuest.

Wirth, Linda

2002. Romper el techo de cristal. Las mujeres en puestos de dirección. Colección Informes OIT 58. Madrid: Ministerio de Trabajo y Asuntos Sociales.

\section{Notas}

A) Explotación de la base de datos de los establecimientos alojativos turísticos asociados a Ashotel según el sexo de la persona que dirige dichos establecimientos, $(n=192)$. B) Realización de encuesta a una muestra de hombres y mujeres en los puestos de responsabilidad en el sector (segregación vertical), $(n=20)$. C) Análisis en perspectiva de género sobre el Convenio Colectivo de Hostelería de la provincia de Santa Cruz de Tenerife, para localizar medidas que favorecen o dificultan el acceso de las mujeres a los puestos de responsabilidad. E) Campo de entrevistas semiestructuradas a agentes claves representantes de entidades vinculadas al sector hotelero: Ashotel, Asociación de gobernantas de hoteles y otras entidades de Tenerife (ASGOTE), Secretaría de la mujer de USO y área de Turismo, Planificación e Investigación del Cabildo de Tenerife (n=4 (3M-1H). D) Entrevistas en profundidad al estilo historias de vida a las mujeres y hombres con puestos de responsabilidad en los hoteles de Tenerife $(n=27)$.

2 La muestra correspondiente a las entrevistas en profundidad a personas en puestos de responsabilidad incluyó 14 Mujeres entrevistadas: directora (2), subdirectora (1), jefa de recepción (1), responsable de reservas (2), jefa de relaciones públicas (1), asistente de dirección (1), subgobernanta (2), gobernanta (2), responsable de compras del hotel (1) y Gex experience manager (1) (equivalente a responsable de calidad, pero con otras muchas funciones asignadas) y 13 hombres entrevistados: director (2), subdirector (1), jefe de recepción (1), jefe de mantenimiento (1), jefe de cocina (2), $2^{\circ}$ jefe de cocina (1), maître (1), $2^{\circ}$ maître (1), director de alimentos y bebidas (2), jefe de actividades (1).

Recibido: 